\title{
Asif's Equation of Charge Variation and Special Relativity
}

\author{
Asif Ali Laghari \\ (Department of Electronics Engineering, Mehran University of Engineering and Technology, Pakistan)
}

\begin{abstract}
The theory of special relativity plays an important role in the modern theory of classical electromagnetism. Considering deeply the effect of Special relativity in Electromagnetism, when a charge particle moves with high speed as comparable to the speed of light in vacuum tube or in space under influence of electromagnetic field, its mass varies under Lorentz transformation [1].The question arises that does its charge vary under Lorentz transformation? In this paper, Asif's equation of charge variation demonstrates the variation of electric charge under Lorentz transformation. The more sophisticated view of electromagnetism expressed by electromagnetic fields in moving inertial frame can be achieved by considering some relativistic effect including charge as well. One can easily achieve the mass-energy relation from Asif's equation of charge variation as proved in this paper.
\end{abstract}

\section{Introduction}

From a historical point of view, it is evident that Maxwell's equations themselves were precursors to the eventual formulation of special relativity by Albert Einstein in 1905 [2]. Purcell argued that, the sources which create electric field are at rest with respect to one of the reference frames which is moving with constant velocity. Given the electric field in the frame where the sources are at rest, Purcell asked: what is the electric field in some other frame? [3].In this paper, we propose Asif's equation of charge variation that is the modern teaching strategy for developing electromagnetic field theory. Asif's equation solves the mysteries of an electric field, magnetic field, electromagnetic wave and behavior of point charge in inertial and non-inertial frame of references with respect to rest observer. In Section II Asif's equation of charge variation is derived, in section III the proposed equation is used to derive mass energy relation for verification and in section IV we conclude the discussion.

\section{Asif's Equation of Charge Variation}

When a charge moves in electric and magnetic field, it experience electromagnetic force. Consider that a charge $Q$ is moving with the uniforms velocity along y-axis with respect to rest observer and electric and magnetic field are applied externally along $\mathrm{z}$-axis and $\mathrm{x}$-axis respectively as shown in the following figure 1 .

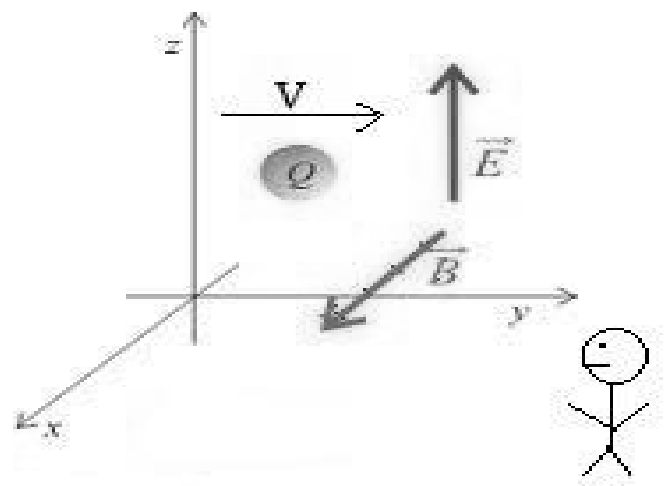

Fig. 1 Charge ' $Q$ ' in moving inertial frame measured by rest observer under the influence of electromagnetic field.

The force experienced by charge due to magnetic field is given by.

$F_{B}=q(V \times B)$

Force due to electric field on a charge is given by.

$F_{E}=q E$

Let us suppose that electric and magnetic field are provided in such a way that electric and magnetic force on a charge becomes equal and charge will deviate due to magnetic field and forms circular path if fluorescent screen is placed in front of it.

$F_{E}=F_{B}$ 
Since magnetic force experienced by charge provides necessary centripetal force which is given by.

$$
\begin{gathered}
F_{B}=F_{c}=\frac{m v^{2}}{r} \\
q v b=\frac{m v^{2}}{r}
\end{gathered}
$$

Solving for linear momentum of charge, above equation can be written as:

$$
p=m v=q B r
$$

Work done on a charge through small distance can be determined by using integral as:

$k \cdot E=\int F \cdot d s$

According to Newton's second law of motion, $F=\frac{d p}{d t}$ where ' $p p^{\prime}$ is linear momentum.

$$
\begin{aligned}
& \text { k. } E=\int \frac{d p}{d t} \cdot d s=\int \frac{d s}{d t} d p \\
& k . E=\int v \cdot d p
\end{aligned}
$$

From equation (2) putting value of $p=q B r$ in above equation. Since ' $B^{\prime}$ and ' ${ }^{\prime}$ ' are constant term.

$$
k \cdot E=B r \int v d(q)
$$

Since charge ' $q^{\prime}$ is variable and depends upon velocity ${ }^{\prime} v^{\prime}$. Therefore according to rule of integration we can equate above equation as.

$$
k \cdot E=B r \int v d(q)=B r \int d(q v)
$$

Now using product rule of derivative and multiplying and dividing by ${ }^{\prime} v^{\prime}$. We obtain:

$k \cdot E=\int \frac{(v d q+q d v) v}{v}$

Since charge is moving with the high speed comparable to the speed of light, to achieve desired relation for charge variation, Lorentz factor is applied [4] which is given by.

$\gamma=\frac{1}{\sqrt{1-\frac{v^{2}}{c^{2}}}}$

Squaring both sides and by cross multiplying Lorentz factor, we have:

$$
\begin{aligned}
& \gamma^{2}\left(1-\frac{v^{2}}{c^{2}}\right)=1 \\
& \gamma^{2} c^{2}-\gamma^{2} v^{2}=c^{2}
\end{aligned}
$$

Taking differential.

$2 \gamma c^{2} d \gamma-2 \gamma v^{2} d \gamma-2 v \gamma^{2} d v=0$

$c^{2} d \gamma=v^{2} d \gamma+v \gamma d v$

$c^{2} d \gamma=(v d \gamma+\gamma d v) v$

In special theory of relativity, small change in the Lorentz factor causes due to mass variation, length contraction or time dilation [5], let us assume that change in the Lorentz factor causes due to charge variation for rest observer in electromagnetic field. Hence replacing $d \gamma$ by $d q$ and $\gamma$ by $q_{3}$ we get:

$c^{2} d q=(v d q+q d v) v$

Or

$$
(v d q+q d v) v=c^{2} d q
$$

Substituting equation (4) in equation (3), we obtain:

$k . E=\frac{B r}{v} \int c^{2} d q$

From equation (2), relation for kinetic energy will be as: 
$K . E=\frac{1}{2} m v^{2}=\frac{1}{2} q v B r$

Hence equation (5) can be written as:

$\frac{1}{2} q v B r=\frac{B r}{v} \int c^{2} d q$

$\frac{1}{2} q v^{2}=\int c^{2} d q=\int v(v d q+q d v)$

Or

$\int c^{2} d q=\int v(v d q+q d v)$

Multiplying both sides by ' $2 q^{\prime}$ and taking right hand side to left hand side. We get:

$\int 2 q c^{2} d q-\int\left(2 q v^{2} d q+2 v q^{2} d v\right)=0$

According to product rule of derivative.

$\int\left(2 q v^{2} d q+2 v q^{2} d v\right)=\int d\left(q^{2} v^{2}\right)$

Hence equation (6) becomes:

$\int 2 q c^{2} d q-\int d\left(q^{2} v^{2}\right)=0$

Now integrating both sides.

$q^{2} c^{2}-q^{2} v^{2}=C$

Where ' $\mathrm{C}$ ' is the constant of integration. To find value of $\mathrm{C}$, we consider that at initial state, velocity of charge is zero. Put $v=0$ in equation (7). We obtain:

$C=q_{0}{ }^{2} c^{2}$

Where ' $q_{0}{ }^{\prime}$ is rest charge because of velocity is zero at initial state.

Substituting value of ${ }^{x} C^{\prime}$ in equation (7), finally we get:

$q^{2} c^{2}-q^{2} v^{2}=q_{0}{ }^{2} c^{2}$

$\frac{q^{2}\left(c^{2}-v^{2}\right)}{c^{2}}=q_{0}{ }^{2}$

$q^{2}\left(1-\frac{v^{2}}{c^{2}}\right)=q_{0}{ }^{2}$

Taking square root on both sides and solving for ' $q$ '.

$q=\frac{q_{0}}{\sqrt{1-\frac{v^{2}}{c^{2}}}}$

It is called Asif's Equation of Charge Variation. Where ' $q$ ' is relativistic charge and ${ }^{\prime} q_{0}{ }^{\prime}$ is rest charge.

\section{Derivation of $E=m c^{2}$ From Asif's Equation of Charge Variation}

From equation (5), as charge starts moving from rest it varies from ' $q_{0}{ }^{\prime}$ to ' $q^{\prime}$ with respect to rest observer.

k. $E=\frac{B r}{v} \int_{q_{0}}^{q} c^{2} d q$

$K . E=\frac{B r q c^{2}}{v}-\frac{B r q_{0} c^{2}}{v}$

Form equation (2), $q B r=m v$ and $q_{\circ} B r=m_{0} v$. Therefore, 
$K . E=\frac{m v c^{2}}{v}-\frac{m_{o} v c^{2}}{v}$
$K . E=m c^{2}-m_{\circ} c^{2}$

The kinetic energy is the difference between relativistic mass energy and rest mass energy [6]. Therefore, total relativistic energy is the sum of the kinetic energy and the rest energy as given by:

$E=m c^{2}$

\section{CONCLUSION}

In this paper we have presented the equation of charge variation so that to treat with the problem of behaviour charges in different frames. The proposed equation shows that in SI units, "charge" is also a variable quantity and depends upon velocity of charge relative to an observer. Then paper discusses about the important consequence of special relativity that is mass-energy relation. Considering this equation, the basic of relativistic electromagnetism and electromagnetic theory can be well established.

\section{REFERENCES}

[1]. Einstein, "On the Electrodynamics of Moving Bodies (1905)," in A. Einstein and F. A. Davis, The Principle of Relativity, London, Dover, 1952

[2]. J. C. Maxwell, "A Dynamical Theory of the Electromagnetic Field (1865)," in W. D. Niven (ed.), The Scientific Papers of James Clerk Maxwell, New York, Dover.

[3]. Purcell, Edward M. “Electricity and magnetism”. Cambridge University Press, 1985.

[4]. Yuen, C.K “ Lorentz transformation of thermodynamics quantities”. American Journal of Physics, 1970.

[5]. A. Einstein, Relativity - "The Special and the General Theory". New York, Crown, 1961.

[6]. Frank J. Blatt.” Modern Physics”. United State of America Press. 1992, pp. 34-35 